Br Heart $\mathcal{F}$ 1985; 53: 123-9

\title{
Assessment of cardiac output by the Doppler ultrasound technique alone
}

\author{
NEVA E HAITES, * FIONA M MCLENNAN, † DONALD H R MOWAT, $\dagger$ JOHN M RAWLES* \\ From the ${ }^{\star}$ Department of Medicine, University of Aberdeen, and the $†$ Aberdeen Maternity Hospital, Aberdeen
}

SUMMARY The normal range of aortic blood velocity was established in 140 healthy adults, using a non-invasive Doppler ultrasound technique. Integration of the area under the velocity-time curve for each heart beat gave stroke distance, which, when multiplied by heart rate, gave minute distance. Stroke distance and minute distance are an indication of stroke volume and cardiac output respectively and both show a progressive decline with age of about $1 \%$ per annum of adult life. Stroke distance gave the greatest discrimination between patient groups and, compared with age corrected normal values, was increased by $43 \%$ in 12 pregnant women, normal in 16 patients convalescing after myocardial infarction, and decreased by $14 \%$ in 15 patients with hypertension, by $31 \%$ in 12 patients with atrial fibrillation, and by $43 \%$ in 13 patients with cardiac failure.

Measurement of aortic blood velocity and its derivatives alone, without determination of aortic size, is a safe, simple, and physiologically valid way of assessing cardiac output.

Cardiac output is the ultimate expression of cardiovascular function, yet we learn little about either from clinical examination. Even the physical signs attributed to cardiac failure are not pathognomonic of impaired cardiac function but may also be found when the heart is functioning normally with volume overload of the circulation. In a whole variety of clinical situations there is therefore a need to be able to assess cardiac output, but until now there has not been a satisfactory non-invasive method.

The Doppler ultrasound technique offers the possibility of a simple to use, non-invasive bedside method of measuring cardiac output, giving absolute values of blood flow for a modest capital outlay and low running cost. Blood velocity is determined ultrasonically at a point in the circulation where the cross sectional area may be measured. Three sites have been described: the mitral valve orifice, ${ }^{1}$ the root of the aorta, ${ }^{2}$ and the aortic arch. ${ }^{34}$ Integration of the area under the velocity-time curve for each heart beat gives a distance that, if multiplied by the cross sectional area at the point of measurement of velocity, gives stroke volume. When the measurements are made in the aorta there are several assumptions implicit in the

Requests for reprints to Dr J M Rawles, Department of Medicine, Aberdeen Royal Infirmary, Foresterhill, Aberdeen AB9 2ZB.

Accepted for publication 2 August 1984 calculation: non-turbulent flow with a flat velocity profile, constant aortic diameter throughout systole, and total stroke volume passing the point of velocity measurement. If velocity is measured in the aortic root no allowance is made for flow to the coronary arteries or to the head and neck if the measurement is made in the aortic arch. The most difficult part technically is measurement of aortic cross sectional area, but even with satisfactory measurements volumetric cardiac output may be considerably overestimated. 5 .

The technique we describe uses blood velocity measurements alone without estimation of cross sectional area. The arch of the aorta is used, where a flat velocity profile is a reasonable assumption, ${ }^{6}$ and blood flow is less likely to be turbulent than in the aortic root, ${ }^{7}$ although a substantial and unknown proportion of stroke volume has left the aorta by the time it reaches the point where velocity is measured. Clinical experience suggests, however, that measuring the cross sectional area is unnecessary for converting the velocity-time integral into a volume, the velocity measurements by themselves closely reflecting cardiac output. This is fortunate as velocity measurements may be made with ease even in very sick patients, whereas measurement of the cross sectional areas of either the aorta or mitral valve may be technically difficult in a substantial proportion of subjects. If velocity measurements are used by themselves to assess 
cardiac output none of the assumptions mentioned above need be made. Velocity alone may be measured with equipment used by relatively unskilled staff whereas if combined with measurement of cross sectional area it is best done with a cross sectional echocardiograph with a Doppler mode. Such equipment is commercially available and may give accurate values for volumetric cardiac output ${ }^{89}$ but is much more expensive and less portable than instruments measuring velocity alone, which have been used in conjunction with echocardiography. ${ }^{1011}$ Considerable skill is required to get good quality cross sectional echocardiograms for the measurement of cross sectional areas, and, because the equipment is multipurpose, it is less likely to be available for repeated measurements at the bedside of a sick patient.

Reproducibility of the technique of transcutaneous aortovelography described here is good, with a 6.6\% deviation from exact agreement with one operator and one of $7.3 \%$ between operators. ${ }^{12}$ While a satisfactory study showing correlation between aortovelography and cardiac output measurement has yet to be reported, several comparisons have been made between aortovelography and cardiac output measured by thermodilution or dye dilution, which show close proportionality between changes in aortovelographic variables and changes in cardiac output. ${ }^{13-15}$

The safety and ease of use of the technique of transcutaneous aortovelography makes the assessment of cardiac output feasible in a wide range of situations, but validation is needed by direct comparison between aortovelography measurements and cardiac output and by performing aortovelography in patients known to have normal and abnormal cardiac outputs. We have previously reported the range of aortic blood velocity in normal subjects and shown that it is independent of sex, body surface area, and blood pressure but declines progressively with age. ${ }^{16}$ We report results from normal subjects, pregnant women, and patients with hypertension, myocardial infarction, atrial fibrillation, or cardiac failure.

\section{Subjects and methods}

NORMAL SUBJECTS

One hundred and seventy five apparently healthy staff and patients attending a general practice surgery were initially selected, but 35 were excluded after a medical history, physical examination, and electrocardiography because of known or suspected heart disease, hypertension, thyro:d disease, concurrent drug treatment, or recent intercurrent disease. The remaining 140 subjects ranged in age from 16 to 74 years with a balanced representation of all ages and both sexes.

\section{PREGNANT WOMEN}

This group comprised 12 healthy women with a mean duration of pregnancy of 18.6 weeks and a mean age of 29 years.

\section{PATIENT GROUPS}

Hypertension-In the search for normal subjects 15 patients with known or newly diagnosed essential hypertension were identified (mean age 53 years). Their mean blood pressure was $165 / 103 \mathrm{~mm} \mathrm{Hg}$ compared with the age corrected value of $136 / 83$ $\mathrm{mm} \mathrm{Hg}$ in the normal subjects. Six were receiving no treatment, five were taking beta blockers, two with the addition of a diuretic, and four were taking a diuretic alone.

Cardiac failure - Thirteen patients with cardiac disease were studied (mean age 57 years) who were admitted to hospital with clinical signs of right, left, or biventricular failure for which they were still requiring treatment.

Myocardial infarction-Sixteen patients recuperating from acute myocardial infarction within the previous 10 days were studied shortly before discharge from hospital. None had clinical evidence of cardiac failure at the time of aortic blood velocity measurements. The mean left ventricular ejection fraction of èight patients from this group was normal at 0.62 when measured at rest by a radionuclide method.

Atrial fibrillation-Twelve patients (mean age 64 years) were studied: three had mitral valve disease, five ischaemic heart disease or hypertension, one treated thyrotoxicosis, and three unexplained atrial fibrillation. None was taking diuretics or had a history of cardiac failure, eight were taking digoxin, and one amiodarone for the control of ventricular rate.

\section{AORTIC BLOOD VELOCITY MEASUREMENT}

In subjects in sinus rhythm aortic blood velocity was recorded over 10 cardiac cycles using the Transcutaneous Aortic Velograph (TAV) Type 1006 (Muirhead Medical Ltd), a restyled version of the equipment described by Sequeira and colleagues. ${ }^{13}$ The recording was made with the subject supine after five minutes' rest. Longer recordings of up to 200 complexes were recorded from patients with atrial fibrillation as part of another study.

A transducer, emitting continuous wave ultrasound at $2 \mathrm{MHz}$, is held in the suprasternal notch and directed at the arch of the aorta. The same transducer detects back scattered ultrasound which generates Doppler shift frequencies proportional to the velocities of blood in the ultrasound pathway. The results of spectral analysis of the Doppler frequencies are displayed on a paper trace, which may be directly calibrated as aortic blood velocity against time. Angulation of the transducer is not critical, and help in 


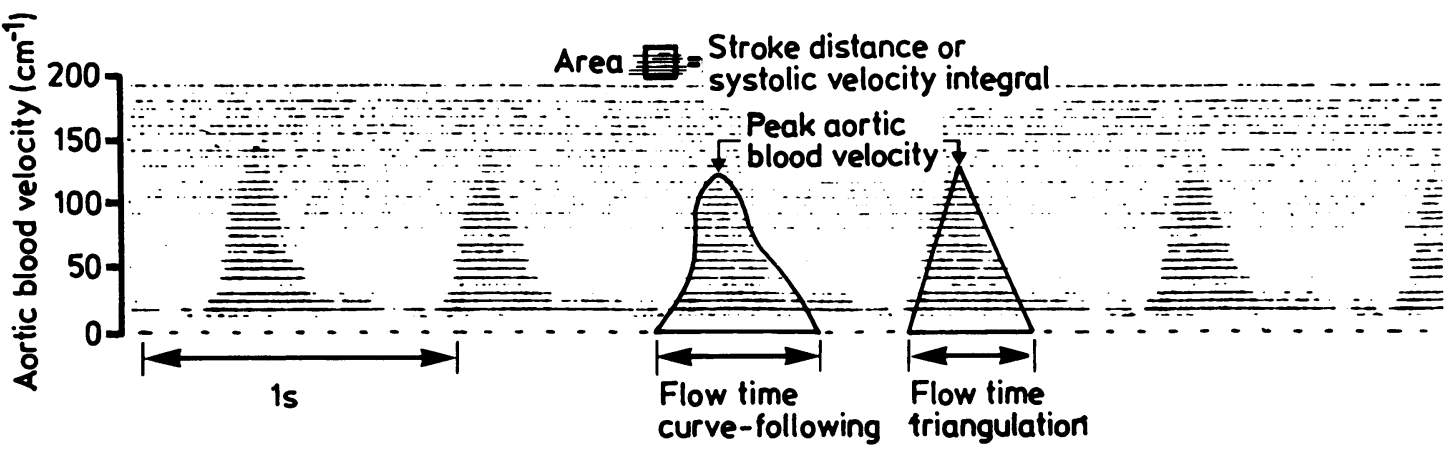

Fig. 1 A typical aortovelography trace showing measurement of stroke distance by curve-following and triangulation.

positioning it is obtained by maximising the pitch of the Doppler frequencies played through a loudspeaker, at which point the ultrasound beam is in line with the direction of blood flow.

\section{INTERPRETATION OF VELOCITY MEASUREMENT}

Fig. 1 shows a typical trace. The outline of the velocity-time complex represents the highest velocity recorded in the ultrasound path at any instant. The complexes are approximately triangular, and the apex represents peak aortic blood velocity occurring in aortic midstream in midsystole, and the length of the base of the triangle represents the duration of left ventricular ejection, or flow-time. The area within the velocity-time complex is dimensionally a distance and is recorded in centimetres as "stroke distance," analogous to stroke volume. Stroke distance multiplied by heart rate in beats per minute gives "minute distance," analogous to cardiac output. The velocitytime complex is not exactly triangular, with some rounding of the apex and non-linearity of leading and trailing edges. In our normal subjects we compare two methods of calculating stroke distance, by measuring the base and height of a superimposed best fit triangle, and by a curve-following programme with a digitiser and computer. Triangulated measurements of stroke distance were used in patients with atrial fibrillation and their controls; in other groups the results were calculated by curve-following. Measurements from 10 consecutive complexes were averaged in sinus rhythm, but up to 200 complexes were averaged in atrial fibrillation.

\section{STATISTICAL METHODS}

Blood velocity measurements in five patient groups were compared with predicted means and their residual standard deviations, obtained from the normal series by using the mean ages of the patient groups in the linear regression equations for stroke distance and minute distance against age. Unpaired $t$ tests were used for comparison of means which are given with 1 standard deviation.

\section{Results}

\section{STROKE DISTANCE BY TRIANGULATION AND CURVE-FOLLOWING}

In 140 healthy subjects the mean peak aortic blood velocity was $102 \mathrm{~cm} \mathrm{~s}^{-1}$ as measured by a curvefollowing programme with a digitiser. This was overestimated by $14 \%$ by triangulation, but triangulation slightly underestimates stroke distance, with mean stroke distances of 19.4 and $19.7 \mathrm{~cm}$ by triangulation and curve-following respectively. For practical purposes either method may be used to measure stroke distance, depending on the availability of digitising equipment, the close agreement between these two methods of measurement being shown in Fig. 2 .

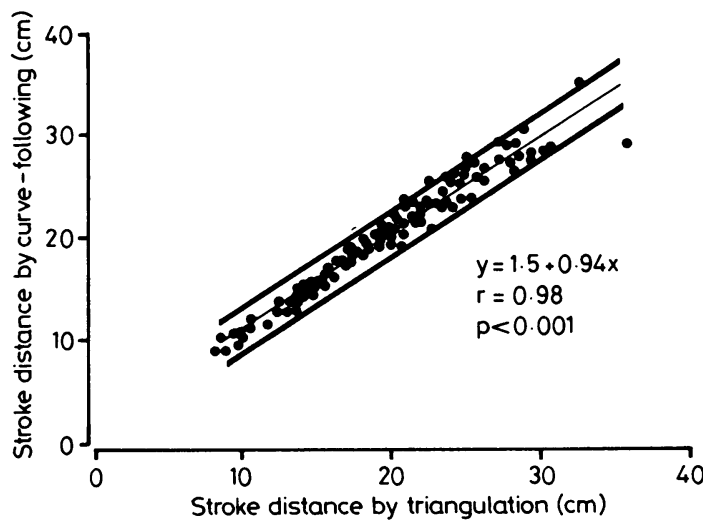

Fig. 2 Stroke distance measured by curve-following and by triangulation. The regression line and $95 \%$ confidence limits are given for data from 140 normal subjects. 


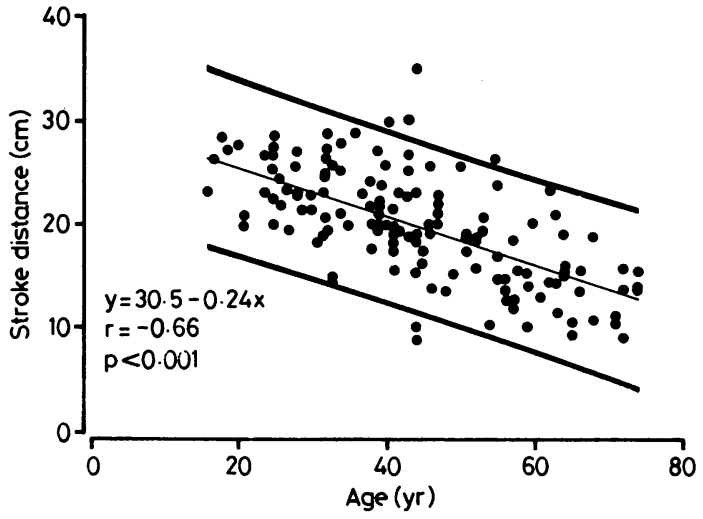

Fig. 3 Regression of stroke distance on age. The regression line and $95 \%$ confidence limits are given for data from 140 normal subjects.

\section{Normal subjects}

Figs. 3 and 4 show the $95 \%$ confidence limits for the decline of stroke distance and minute distance with age. Both measurements fall by about $45 \%$ between the ages of 20 and 70 years. The systolic-velocity integral or stroke distance is highly correlated with peak aortic velocity $(r=0.91, p<0.001)$ but not with flow-time $(r=0.08,0.02<p<0.4)$, indicating that in normal subjects changes in stroke distance are brought about by variation in blood velocity rather than in ejection time.

\section{Patient groups}

Fig. 5 shows the mean values of stroke distance and minute distance in pregnancy, hypertension, cardiac failure, myocardial infarction, and atrial fibrillation compared with age corrected normal values. Peak aortic blood velocity and flow time, the apex and base of

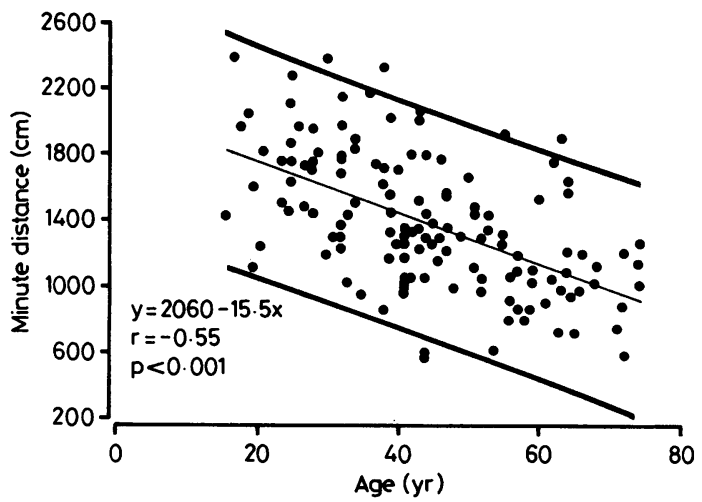

Fig. 4 Regression of minute distance on age. The regression line and $95 \%$ confidence limits are given for data from 140 normal subjects. the triangular velocity-time complex, though not correlated with each other in normal subjects, are nevertheless altered in the same direction in different patient groups. Stroke distance, the area of the complex, being closely related to the product of peak aortic blood velocity and flow-time, shows a greater separation between groups than either of these measurements. Since a reduced stroke volume or stroke distance will lead to an increased heart rate and a proportionately smaller reduction in cardiac output minute distance therefore shows less discrimination between groups than stroke distance.

Pregnancy -In pregnancy there is a $43 \%$ increase in stroke distance $(p<0.001)$ resulting from a $30 \%$ increase in peak aortic blood velocity $(p<0.001)$ and a $12 \%$ increase in flow-time $(p<0.01)$. Minute distance shows an even greater increase over normal at $56 \%$ $(p<0.001)$ since mean heart rate is five beats per minute faster in pregnancy $(0.1<\mathrm{p}<0.2)$.

Hypertension-Although neither peak aortic blood velocity or flow time are significantly reduced, stroke distance and minute distance are $13 \%$ and $15 \%$ lower

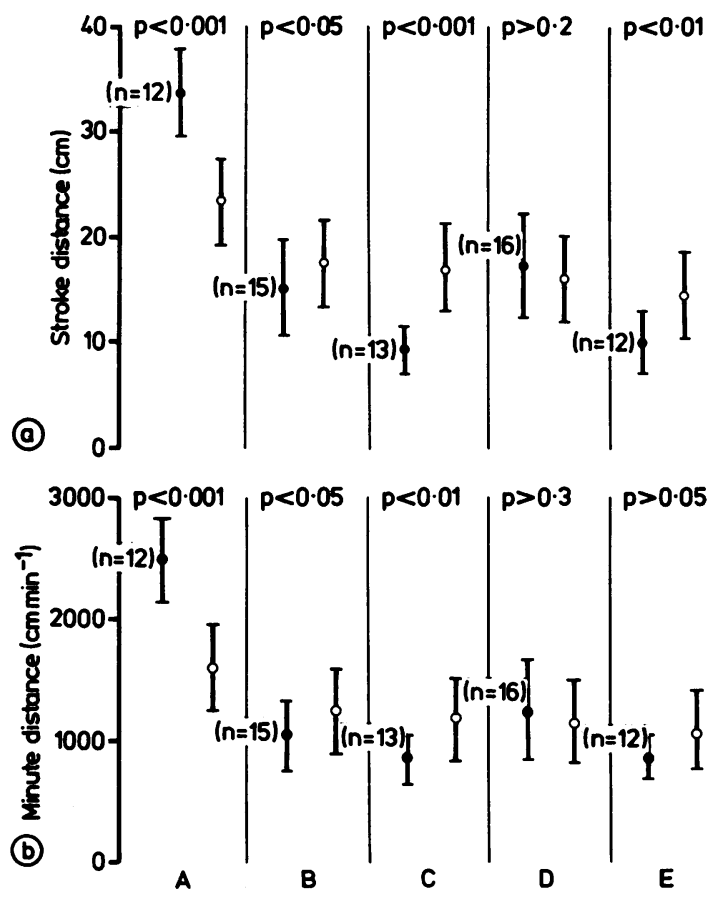

Fig. 5 (a) Mean (SD) stroke distance and (b) mean (SD) minute distance compared with age corrected normal (O) values $(n=140)$ in five patient groups (O): (A) pregnancy (mean age 29 yr); (B) hypertension (mean age 53 yr); (C) cardiac failure (mean age $57 \mathrm{yr}$ ); (D) myocardial infarction (mean age $60 \mathrm{yr}$ ); (E) atrial fibrillation (mean age $64 \mathrm{yr}$ ). 
than normal respectively $(p<0.05)$. The number of subjects in this group is too small to enable us to say whether this reduction in cardiac output is due to hypertension itself or its treatment.

Cardiac failure-Peak aortic blood velocity and flow time are reduced by $23 \%$ and $25 \%$ leading to $43 \%$ reduction in stroke distance. Heart rate is significantly increased by 20 beats per minute to 90 beats/min $(p<0.001)$, compensating to some extent for the reduced stroke distance, minute distance being reduced by $28 \%$.

Myocardial infarction-Aortic blood velocity and its derivatives were normal in this group of convalescent patients.

Atrial fibrillation-Peak aortic blood velocity and flow time are both reduced by $17 \%$ leading to $31 \%$ reduction in stroke distance. Ventricular rate is significantly increased to 87 beats per minute $(\mathrm{p}<0.001)$, compensating partially for the reduced stroke distance, minute distance being reduced but not significantly.

\section{Discussion}

Cardiac output is the ultimate expression of cardiovascular function, so it is surprising that its measurement is made so seldom in clinical practice. This relates to difficulties of measurement but also to its interpretation. Volumetric cardiac output has to be corrected for variation in body size and by convention this is done by division with an estimate of body surface area, even though the statistical basis for this manoeuvre is suspect, and the correction breaks down in neonates. ${ }^{17}$ Cardiac output diminishes with age, ${ }^{18}$ but there are no generally accepted normal values which take age into consideration.

Another problem is that homeostatic mechanisms seek to stabilise cardiac output, the most important of these being alteration of heart rate. Cardiac output per beat, or stroke volume may therefore be a more fundamental expression of cardiovascular function, and that too is usually corrected for body size by division by body surface area. By dimensional analysis, volume/area $=$ distance and stroke volume/body surface area =stroke volume index, the latter therefore being a linear measurement of distance.

Non-invasive measurement of cardiac output by combined Doppler and echocardiography depends on measurement of the systolic velocity integral, or stroke distance, and the cross sectional area at the point where the velocity measurement is made. The systolic velocity integral is then multiplied by the cross sectional area to give stroke volume. The relation between stroke volume and stroke distance measured in the aorta is thus approximately as follows: stroke volume/aortic cross sectional area $=$ stroke distance. Put in this way we see the close analogy between stroke volume index and stroke distance. Both are unidimensional and corrected for variation in body size, the former by body surface area and the latter by aortic cross sectional area, which may reflect body size and metabolic requirements rather better than body surface area. Having measured stroke distance directly we consider it unnecessary to convert it to a volumetric measurement by multiplication with aortic cross sectional area only to correct for body size by dividing arbitrarily by another area, that of the body surface.

If stroke distance is biologically corrected for body size there should be no correlation with body surface area, which is indeed the case, ${ }^{16}$ and aortic blood velocity might be expected to have a similar value throughout infancy and childhood, which is also true, ${ }^{19}$ which cannot be said of the cardiac index. ${ }^{20} 21$

These results indicate that once a normal range of values is established, aortic blood velocity measurements may be used by themselves to assess cardiac output without conversion to a volumetric measurement or correction for body size. The pronounced decline of aortic blood velocity and its derivatives with age means that age correction is necessary, however, but prediction of mean values at any adult age under 75 years is readily done from our data using the regression equations relating velocity measurements to age.

There are two possible reasons for the reduction of aortic blood velocity and its derivatives with age: reduction of cardiac output and enlargement of the aorta. Volumetric cardiac output decreases by about $1 \%$ per annum between the ages of 20 and 80,18 a comparable figure to the decline of minute distance of $0.88 \%$ a year in our series. Furthermore, during adult life there may be some passive dilatation of the aorta, but evidence on this point is scanty. One source reports an increase in external aortic diameter of $0.75 \%$ a year. ${ }^{22}$

In pregnancy, an increase in both stroke volume and heart rate contribute to an increased cardiac output of about $40 \%$ in mid-trimester. ${ }^{23}$ Our finding of a $56 \%$ increase in minute distance is a higher percentage increase than has been recorded for cardiac output, but we have confirmed this finding in a separate group of patients at an earlier stage of pregnancy.

In the early stages of hypertension cardiac output may be raised, but later with increasing peripheral resistance it falls below normal. ${ }^{24}$ Treatment either with beta blockers or diuretics may also cause a reduction of cardiac output, 2526 so our finding of a significant reduction of minute distance in the hypertensive group is not unexpected.

The definition of cardiac failure is elusive, ${ }^{27}$ but it has long been known that cardiac output is reduced in 
the syndrome of congestive cardiac failure, although there is some overlap with the range of values found in normal subjects. ${ }^{28}$ In our patients with cardiac failure, the mean values of aortic blood velocity and its derivatives were all significantly reduced. Stroke distance showed the greatest percentage reduction, an increased heart rate providing partial compensation leading to a smaller proportional fall in minute distance. As might be expected, the range of values for stroke distance found in cardiac failure overlapped with normal age corrected values, but its measurement gives an indication of the severity of cardiac dysfunction that is not available from the clinical examination.

At the time of discharge all aortic blood velocity measurements were normal in the patients with myocardial infarction that we studied, who had had an uncomplicated course with no clinical evidence of cardiac failure and, where measured, normal left ventricular ejection fractions. Stroke volume and cardiac output have been measured in patients with myocardial infarction, ${ }^{29}$ and while stroke volume and often cardiac output were diminished on the first days after infarction, both were normal by the time of discharge from hospital.

In atrial fibrillation, loss of atrial function leads to reduction of stroke volume and cardiac output. ${ }^{30} 31$ Our results show a $31 \%$ reduction of stroke distance in atrial fibrillation with a $19 \%$ reduction of minute distance, which does not, however, achieve statistical significance. The increased ventricular rate in atrial fibrillation compensates for the fall in stroke volume and may well be under automatic control, especially in patients taking digoxin. ${ }^{32}$ Measurement of cardiac output in atrial fibrillation could bring a new precision to the control of the ventricular rate: the optimum rate is that which results in a normal cardiac output.

The non-invasive assessment of cardiac output by aortic blood velocity measurements by themselves has given results that are wholly consistent with previously published work where cardiac output has been measured by conventional invasive techniques. Because of the safety and acceptability of the method it is possible to collect a large series of results from normal subjects to compare with those from patients of any age, in whom it is now feasible to make frequent sequential measurements at the bedside or in the outpatient clinic. Cardiac output is such an important haemodynamic variable that its more frequent measurement-and a greater understanding of the factors that influence it-must lead to improved patient care.

\section{References}

1 Fisher DC, Sahn DJ, Friedman MJ, et al. The mitral valve orifice method for noninvasive two-dimensional echo Doppler determinations of cardiac output. Circulation 1983; 67: 872-7.

2 Huntsman LL, Stewart DK, Barnes SR, Franklin SB, Colocousis JS, Hessel EA. Noninvasive Doppler determination of cardiac output in man. Clinical validation. Circulation 1983; 67: 593-602.

3 Light H. Transcutaneous aortovelography. A new window on the circulation? Br Heart $\mathcal{F}$ 1976; 38: 433-42.

4 Rawles JM, Haites NE. Doppler ultrasound measurement of cardiac output: transcutaneous aortovelography. Br 7 Hosp Med 1984; 31: 292-7.

5 Ihlen H, Amlie JP, Dale J, et al. Determination of cardiac output by Doppler echocardiography. Br Heart $\mathcal{F}$ 1984; 51: 54-60.

6 Schultz DL, Tunstall-Pedoe DS, Lee G de J, Gunning AJ, Bellhouse BJ. Velocity distribution and transition in the arterial system. In: Wolstenholme GEW, Knight J, eds. Circulatory and respiratory mass transport. London: J \& A Churchill, 1969: 172-202.

7 Light LH, Cross G. Convenient monitoring of cardiac output and global left ventricular function by transcutaneous aortovelography - an effective alternative to cardiac output measurements. In: Spencer MP, ed. Cardiac Doppler diagnosis. The Hague: Martinus Nijhoff, 1983: 69-80.

8 Fisher DC, Sahn DJ, Friedman MJ, et al. The effect of variations on pulsed Doppler sampling site on calculation of cardiac output: an expermental study in open-chest dogs. Circulation 1983; 67: 370-6.

9 Magnin PA, Stewart JA, Myers S, Von Ramm O, Kisslo JA. Combined Doppler and phased-array echocardiographic estimation of cardiac output. Circulation 1981; 63: 388-92.

10 Loeppky, Greene ER, Hoekenga DE, Caprihan A, Luft UC. Beat-by-beat stroke volume assessment by pulsed Doppler in upright and supine exercise. $\mathrm{f}$ Appl Physiol 1981; 50: 1173-82.

11 Alverson DC, Eldridge $M$, Dillon $T$, Yabek SM, Berman W Jr. Non-invasive pulsed Doppler determination of cardiac output in neonates and children. $\mathcal{F}$ Pediatr 1982; 101: 46-50.

12 Fraser CB, Light LH, Shinebourne EA, Buchtal A, Healy MJR, Beardshaw JA. Transcutaneous aortovelography: reproducibility in adults and children. Eur $\mathcal{F}$ Cardiol 1976; 4: 181-9.

13 Sequeira RF, Light LH, Cross G, Raftery EB. Transcutaneous aortovelography. A quantitative evaluation. Br Heart F 1976; 38: 443-50.

14 Light LH, Sequeira RF, Cross G, Bilton A, Hanson GC. Flow-orientated circulatory patients assessment and management using transcutaneous aortovelography. f Nucl Med Allied Sci 1979; 23: 137-44.

15 Distante A, Moscarelli E, Rovai D, L'Abbate A. Monitoring changes in cardiac output by transcutaneous aortovelography a non-invasive Doppler technique: comparison with thermodilution. $\mathcal{F}$ Nucl Med Allied Sci 1980; 24: 171-5.

16 Mowat DHR, Haites NE, Rawles JM. Aortic blood velocity measurement in healthy adults using a simple ultrasound technique. Cardiovasc Res 1983; 17: 75-80.

17 Guyton AC, Jones CE, Coleman TG. Circulatory physiol- 
ogy: cardiac output and its regulation. 2nd ed. Philadelphia: W B Saunders, 1973.

18 Brandfonbrener M, Landowne M, Shock NW. Changes in cardiac output with age. Circulation 1955; 12: 557-66.

19 Sequeira RF. Transcutaneous aortovelography. In: Woodcock JP, Sequeira RF, eds. Doppler ultrasound in the study of the central and peripheral circulation. Bristol: Bristol University Printing Unit, 1978: 30-8.

20 Brotmacher L, Deuchar DC. The systemic blood flow in congenital heart disease, with an examination of the validity of the cardiac index. Clin Sci 1956; 15: 441-56.

21 Jegier W, Sekelj P, Auld PAM, Simpson R, McGregor $M$. The relation between cardiac output and body size. Br Heart f 1963; 25: 425-30.

22 Documenta Geigy. Scientific tables. Macclesfield: Geigy Pharmaceuticals, 1970.

23 Hytten F, Leitch I. The physiology of human pregnancy. 2nd ed. Oxford: Blackwell Scientific Publications, 1971: 69-110.

24 Lund-Johansen P. Central haemodynamics in essential hypertension. Acta Med Scand [Suppl] 1977; 606: 35-42.

25 Conway J, Lauwers P. Hemodynamic and hypotensive effects of long term therapy with chlorothiazide. Circula- tion 1960; $21: 21-7$.

26 Bevegard S, Castenfors J, Danielson M. Haemodynamic effects of saluretic treatment and beta-receptor blockade in patients with essential hypertension. Acta Med Scand 1977; 201: 99-104.

27 Harris P. Evolution and the cardiac patient. 6. Origins of congestive cardiac failure. Cardiovasc Res 1983; 17: 440-5.

28 Stead EA Jr, Durham NC, Warren JV, Brannon ES. Cardiac output in congestive cardiac failure. Am Heart $\mathcal{f}$ 1948; 35: 529-41.

29 Malcrona R. Haemodynamics in myocardial infarction. Acta Med Scand [Suppl] 1964; 417: 1-54.

30 Ferrer MI, Harvey RM, Cathcart RT, Cournand A, Richards DW Jr. Hemodynamic studies in rheumatic heart disease. Circulation 1952; 6: 688-710.

31 Wade G, Werkö L, Eliasch H, Gidlund A, Largerlöf $H$. The haemodynamic basis of the symptoms and signs in mitral valvular disease. $Q \mathcal{F} \mathrm{Med} 1952 ; 21: 361-83$.

32 Ferrari A, Bonazzi O, Gregorini L, Gardumi M, Perondi R, Mancia G. Modification of the baroreceptor control of atrioventricular conduction induced by digitalis in man. Cardiovasc Res 1983; 17: 633-41. 